\title{
Comparative efficacy and safety of late surfactant preparations: a retrospective study
}

Morgan D. Lane ${ }^{1}$, Sujata Kishnani ${ }^{1}$, Obianuju Udemadu ${ }^{1}$, Samuel Eshun Danquah ${ }^{1}$, Robert M. Treadway III ${ }^{1}$, Aaliyah Langman ${ }^{1}$, Stephen Balevic ${ }^{1,2}$, Wesley M. Jackson ${ }^{3}$, Matthew Laughon (D) $^{3}$, Christoph P. Hornik ${ }^{1,2}$, Rachel G. Greenberg (iD) ${ }^{1,2}$, Reese H. Clark (iD ${ }^{4}$ and Kanecia O. Zimmerman (1D ${ }^{1,2}$

(c) The Author(s), under exclusive licence to Springer Nature America, Inc. 2021

OBJECTIVE: Characterize the use, efficacy, and safety of poractant alfa and calfactant surfactants compared to beractant in preterm infants receiving late surfactant.

STUDY DESIGN: We included infants <37 weeks gestational age (GA) discharged from Pediatrix Medical Group-managed neonatal intensive care units (1997-2017). Efficacy and safety outcomes of interest were analyzed.

RESULTS: Of 184,770 infants administered surfactant at any time, 7846 (4.23\%) received late surfactant at a median (25th, 75th percentile) PNA of 8 days (3, 22); specifically, 2976 received poractant alfa (38\%), 2890 beractant (37\%), and 1936 calfactant (25\%). We identified no significant differences in composite efficacy or safety outcomes between surfactants in the primary analysis, but 33-36 week GA infants administered poractant alfa had significantly greater odds of developing a safety event.

CONCLUSIONS: Compared to beractant, there is no evidence of overall superior efficacy or safety of poractant alfa.

Journal of Perinatology (2021) 41:2639-2644; https://doi.org/10.1038/s41372-021-01142-2

\section{INTRODUCTION}

Before exogenous surfactant, $40-50 \%$ of preterm infants died from respiratory distress syndrome (RDS) [1, 2]. During the 1980s, several randomized trials found that three animal-derived surfactants-beractant, calfactant, and poractant alfa-reduced RDS and associated sequelae in preterm infants. Since receiving United States Food and Drug Administration (FDA) approval, use of surfactants in preterm infants has become routine, particularly the use of poractant alfa [3], perhaps encouraged by its high concentration of critical phospholipids that allows administration of smaller drug volumes [4, 5]. Furthermore, evidence suggests decreased need for respiratory support, shorter intubation periods, and reduced mortality in infants with RDS when using poractant alfa versus beractant [6-9].

Whether differences in surfactant safety and efficacy profiles exist when used for rescue, beyond the labeled postnatal age [PNA] of $48 \mathrm{~h}$ for beractant and poractant alfa, and beyond $72 \mathrm{~h}$ for calfactant (i.e., late surfactant), remains unclear [3, 10-12]. Infants receiving late surfactant may be treated to prevent bronchopulmonary dysplasia (BPD), or have refractory RDS or other conditions (e.g., aspiration pneumonitis, pneumonia, and extracorporeal membrane oxygenation) [13-15]. In these cases, the relative safety and efficacy of surfactants may be altered due to varied surfactant compositions and treatment of different disease patterns. We sought to characterize the use, efficacy, and safety profiles of calfactant and poractant alfa relative to beractant, the first FDA-approved surfactant, when used beyond the labeled PNA.

\section{MATERIALS AND METHODS}

\section{Study design}

We included all infants born at $<37$ weeks gestational age (GA) discharged from one of 406 Pediatrix Medical Group-managed neonatal intensive care units (NICUs) (1997-2017). We excluded infants with major congenital malformations, including any conditions that were life-threatening, debilitating, or requiring surgery early in life. We retrieved data from the Pediatrix BabySteps Clinical Data Warehouse by extracting daily progress notes, billing diagnoses, and physician orders [16].

Infants who received two types of late surfactant on the same day were included in the count of late surfactant, but excluded from further analyses.

\begin{abstract}
Definitions
We defined late surfactant as administration in infants receiving at least one dose of beractant, poractant alfa, or calfactant after the PNA recommended on the FDA label [10-12], including those with and without prior receipt of surfactant within the labeled PNA. We defined the following as outcomes: air leak syndrome, BPD, length of mechanical ventilation after late surfactant administration, hemodynamic instability, respiratory deterioration, sepsis, pulmonary hemorrhage, death within 3 days of drug administration, PMA at the time of discharge, and death prior to discharge. We defined air leak syndrome as a diagnosis of pneumothorax or pulmonary interstitial emphysema. We defined BPD according to previously-established criteria (i.e., born at $<32$ weeks GA and
\end{abstract}

\footnotetext{
'Duke Clinical Research Institute, Duke University, Durham, NC, USA. ${ }^{2}$ Department of Pediatrics, Duke University, Durham, NC, USA. ${ }^{3}$ Neonatal-Perinatal Medicine, University of

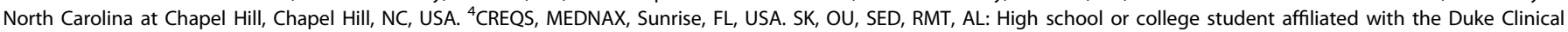
Research Institute's R25 Summer Training in Academic Research (STAR) Program. ${ }^{凶}$ email: kanecia.zimmerman@duke.edu
} 
required continuous oxygen or respiratory support from $36 / 0$ to $37 /$ 0 weeks PMA or born at $\geq 32$ weeks $G A$ and required continuous oxygen or respiratory support from 28 to 34 days after birth) [17]. We defined length of mechanical ventilation as the number of days mechanically ventilated following late surfactant administration. We identified hemodynamic instability by a need for vasopressors on the day of or after surfactant. We defined respiratory deterioration as: (1) an increase in the maximum fraction of inspired oxygen compared to the day of surfactant administration, with the same level of respiratory support, or (2) escalation in the level of respiratory support compared to the day of surfactant administration. From lowest to highest, escalation in respiratory support included any increase to a higher level of oxyhood, nasal cannula, continuous positive airway pressure, conventional ventilator, or high frequency ventilator. We defined sepsis as growth of any organism not typically considered a contaminant on a blood, cerebrospinal fluid, or catheterized urine culture.

\section{Statistical analysis}

We described patient demographics, concomitant diagnoses, outcomes, and surfactant use over time using summary statistics for each surfactant type. Among sites with $>100$ infants meeting inclusion/exclusion criteria, we evaluated the percentage of infants receiving late surfactant use and mean GA by site (Supplementary Fig. 1). We also characterized concomitant diagnoses that might explain reasons for late surfactant administration, including meconium aspiration syndrome, pneumonia, congenital diaphragmatic hernia, pulmonary hemorrhage, requirement for extracorporeal membrane oxygenation, aspiration, BPD, atelectasis, pulmonary edema, respiratory insufficiency, respiratory syncytial virus, and respiratory disease of prematurity.

We used a multivariable logistic regression with random effects for site to evaluate the association between surfactant type and composite efficacy outcome of the absence of air leak syndrome, BPD, or all-cause death prior to discharge. A second multivariable logistic regression model with random effects for site compared the association between surfactant type and the composite safety outcome of respiratory deterioration, hemodynamic instability, pulmonary hemorrhage, sepsis, or death within 3 days after administration. We selected the following covariates a priori based on available literature: antenatal steroid exposure, small for GA status, discharge year (to account for changes in practice over time), sex, race, PNA, and GA. We also evaluated an interaction term between GA and surfactant type to evaluate heterogeneity in safety and efficacy across GAs, to account for known variations in GA for which these drugs are labeled. Based on statistical significance of this interaction term at $p<0.05$ in the safety regression, we performed a stratum-specific analysis to examine whether the composite safety outcome differed by GA group. We reported odds ratios (ORs) and 95\% confidence intervals (Cls) for each model. For the primary analyses, we also investigated fixed effects for site and models without site effects because prior evidence suggested that site variation might lead to statistically significant results that are not otherwise explained [17] (Supplementary Methods). We conducted a sensitivity analysis limited to the infants receiving only late surfactant to account for possible differences between these infants and those who received late surfactant after prior doses within the labeled PNA.

All analyses were pre-specified. We considered $p$ values $<0.05$ to be statistically significant, conducting all analyses using Stata version 16.1 (StataCorp, College Station, TX). The Duke Institutional Review Board approved this study with a waiver of consent.

\section{RESULTS}

\section{Surfactant use over time}

Of 717,960 preterm infants, $184,770(26 \%)$ received surfactant at some time during their NICU stay, and 7846/184,770 (4\%) infants were administered late surfactant. Total surfactant administration steadily decreased starting in 2007, but late surfactant administration increased over time, peaking in 2017 (Figs. 1, 2). Beractant and calfactant were favored until 2011 , when poractant alfa was more commonly used. Use varied greatly by site $(0$ to $>20 \%$ of preterm infants; Fig. 3); 56 (16\%) of centers administered no late surfactant, while $23(6.74 \%)$ centers administered late surfactant to $\geq 2.5 \%$ of preterm infants.

Among those receiving late surfactant, the median (25th, 75th percentile) GA and BW were 28 weeks $(25,32)$ and $1040 \mathrm{~g}(720$,

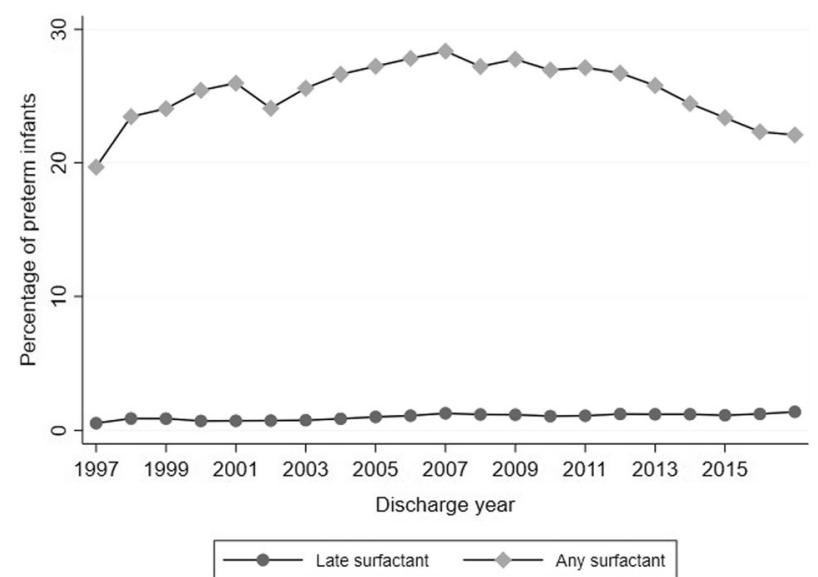

Fig. 1 Surfactant trends. Overall and late surfactant trends.

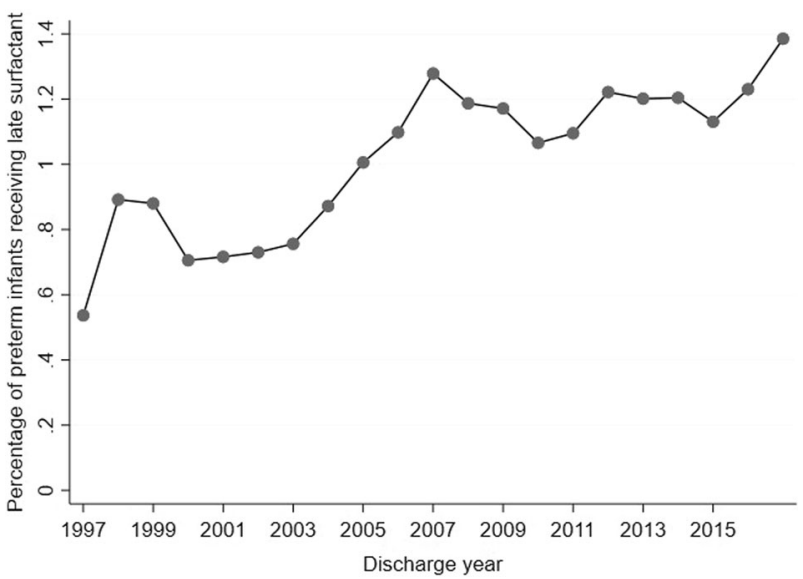

Fig. 2 Administration trend over time. Late surfactant administration trend over time.

1690), respectively. Overall, infants received late surfactant at a median PNA of 8 days $(3,22)$ (Table 1): 2890 (37\%) received beractant, 1936 (25\%) received calfactant, 2976 (38\%) received poractant alfa, and $44(<1 \%)$ received more than one type. Of 4377 infants who received late surfactant and had complete data on all surfactant administrations, $2649(61 \%)$ received surfactant prior to late administration (Supplementary Results). Of infants who received one late surfactant, 6846 (88\%) had been diagnosed with RDS during hospitalization, and 1575 (20\%) had no concomitant diagnosis of interest within the week prior to administration. We did not identify any infants with diagnosis of the following pro-inflammatory states leading to possible surfactant inactivation (meconium aspiration syndrome, pneumonia, congenital diaphragmatic hernia, pulmonary hemorrhage, requirement for extracorporeal membrane oxygenation, aspiration, or pulmonary edema). Infants were also not identified to have atelectasis or other respiratory disease of prematurity. In total, 3677/4565 (81\%) infants were mechanically ventilated on the day prior to surfactant, for a median (25th, 75 th percentile) of $4(3,10)$ days.

\section{Efficacy outcomes}

Of 6442 infants who received one late surfactant and had complete data on the outcomes of death and BPD, 3411 (53\%) were alive without BPD at discharge, including 1495 (62\%) receiving beractant, $654(42 \%)$ receiving calfactant, and 1262 $(51 \%)$ receiving poractant alfa (Table 2 ). According to the 
multivariable model, administration of calfactant or poractant alfa demonstrated no statistically significant difference in the incidence of the composite efficacy outcome compared to beractant, which remained true when fixed effects for site were included in the model (Supplementary Results, Supplementary Table 1).

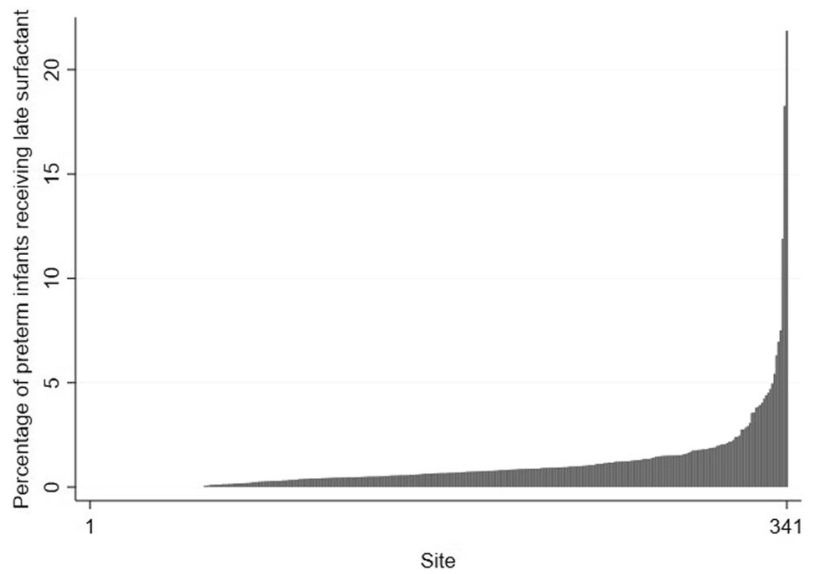

Fig. 3 Administration by site. Late surfactant administration by site.

\section{Safety outcomes}

After late surfactant, 1918 (25\%) infants experienced hemodynamic instability. Of 7557 infants who had complete respiratory support data, 3819 (51\%) experienced respiratory deterioration. Sepsis and pulmonary hemorrhage occurred in 290 (4\%) and 78 (1\%), respectively (Table 3 ). On adjusted analysis, there was no overall statistically significant difference in the composite safety outcome, but when stratified by GA, infants 33-36 weeks GA were significantly more likely to develop a safety outcome if administered poractant alfa compared to beractant (OR 1.58; Cl $1.17,2.16)$. This result remained statistically significant using models with fixed effects for site (OR 1.64; $\mathrm{Cl} 1.04,2.58)$ and models not including site (OR 1.58; $\mathrm{Cl} 1.17,2.16)$ (see Supplementary Tables 2, 3).

\section{Sensitivity analyses}

Sensitivity analysis limited to the population receiving only late surfactant demonstrated no differences in composite efficacy or safety outcomes for infants receiving calfactant or poractant alfa compared to those receiving beractant (Supplementary Tables 4-8).

\section{DISCUSSION}

We successfully characterized the use, efficacy, and safety profile of poractant alfa and calfactant in comparison to the moreestablished beractant, when used after the label-recommended PNA. Over time, overall use of surfactant in preterm infants

Table 1. Infant demographics by surfactant type ${ }^{a}$.

\begin{tabular}{|c|c|c|c|c|}
\hline & $\begin{array}{l}\text { Beractant } \\
N=\mathbf{2 8 9 0}(\%)\end{array}$ & $\begin{array}{l}\text { Calfactant } \\
N=1936(\%)\end{array}$ & $\begin{array}{c}\text { Poractant alfa } \\
N=2976(\%)\end{array}$ & $\begin{array}{l}\text { Total } \\
N=7802 \text { (\%) }\end{array}$ \\
\hline$\leq 25$ & $529(18)$ & $740(38)$ & $941(32)$ & $2210(28)$ \\
\hline $29-32$ & $778(27)$ & $410(21)$ & $694(23)$ & $1882(24)$ \\
\hline $33-36$ & $835(29)$ & $185(10)$ & $586(20)$ & $1606(21)$ \\
\hline $1000-499$ & $696(24)$ & $424(22)$ & $647(22)$ & $1767(23)$ \\
\hline $1500-2499$ & $769(27)$ & $270(14)$ & $576(19)$ & $1615(21)$ \\
\hline$>2499$ & $385(13)$ & $79(4)$ & $274(9)$ & $738(9)$ \\
\hline \multicolumn{5}{|l|}{ Postnatal age (days) } \\
\hline $3-<7$ & $1540(53)$ & $537(28)$ & $1436(48)$ & $3513(45)$ \\
\hline Race/ethnicity & $n=2789$ & $n=1868$ & $n=2858$ & $n=7515$ \\
\hline White & $1428(51)$ & $1007(54)$ & $1499(52)$ & $3934(52)$ \\
\hline African American & $595(21)$ & $463(25)$ & $627(22)$ & $1685(22)$ \\
\hline Hispanic & $678(24)$ & $326(17)$ & $598(21)$ & $1602(21)$ \\
\hline Other & $88(3)$ & $72(4)$ & $134(5)$ & $294(4)$ \\
\hline Prenatal steroids & 1699 (59) & $1433(74)$ & $2129(72)$ & $5261(67)$ \\
\hline PDA ligation & $275(10)$ & $401(21)$ & $366(12)$ & $1042(13)$ \\
\hline Male & $1741(60)$ & $1113 / 1935(58)$ & $1782 / 2974(60)$ & 4636/7799 (59) \\
\hline Received $\geq 1$ prior dose of surfactant ${ }^{b}$ & $821 / 1583(52)$ & $579 / 1019(57)$ & $1205 / 1731(70)$ & $2605 / 4333(60)$ \\
\hline On-label for BW and GA & $1268(44)$ & $1303(67)$ & $1661(56)$ & $4232(54)$ \\
\hline
\end{tabular}

$B W$ Birth weight, GA gestational age, PDA patent ductus arteriosus.

a Infants who were transferred in and had no documentation of prior surfactant were excluded from this analysis.

${ }^{b}$ Includes infants with and without prior receipt of surfactant within the labeled PNA. 
Table 2. Efficacy outcomes by surfactant type ${ }^{a}$.

\begin{tabular}{|c|c|c|c|c|}
\hline GA group count $(\%)$ & $\begin{array}{l}\text { Beractant } \\
\text { Total } \\
\mathbf{N}=\mathbf{2 8 9 0}\end{array}$ & $\begin{array}{l}\text { Calfactant } \\
\text { Total } \\
N=1936\end{array}$ & $\begin{array}{l}\text { Poractant alfa } \\
\text { Total } \\
N=2976\end{array}$ & $\begin{array}{l}\text { Total } \\
\text { Total } \\
\mathbf{N}=\mathbf{7 8 0 2}\end{array}$ \\
\hline No air leak & $2767(96)$ & $1881(97)$ & $2879(97)$ & $7527(96)$ \\
\hline Alive without $\mathrm{BPD}^{\mathrm{b}}$ & $1495 / 2393(62)$ & $654 / 1553(42)$ & $1262 / 2496(51)$ & $3411 / 6442(53)$ \\
\hline Alive at discharge ${ }^{c}$ & $2371 / 2595(91)$ & $1508 / 1714(88)$ & $2409 / 2669(90)$ & $6288 / 6978(90)$ \\
\hline PMA at discharge (completed weeks) & $n=2089$ & $n=1323$ & $n=2240$ & $n=5652$ \\
\hline$<37$ & $827(40)$ & $337(25)$ & $534(24)$ & $1698(30)$ \\
\hline $37-39$ & $821(39)$ & $475(36)$ & $950(42)$ & $2246(40)$ \\
\hline $40-43$ & $315(15)$ & $320(24)$ & $506(23)$ & $1141(20)$ \\
\hline$>43$ & $126(6)$ & $191(14)$ & $250(11)$ & $567(10)$ \\
\hline Length of mechanical ventilation (days) & $n=2848$ & $n=1914$ & $n=2952$ & $n=7714$ \\
\hline$<3$ & $1125(40)$ & $772(40)$ & $1289(44)$ & $3186(41)$ \\
\hline $3-6$ & $148(5)$ & $79(4)$ & $105(4)$ & $332(4)$ \\
\hline $7-13$ & $345(12)$ & $109(6)$ & $180(6)$ & $634(8)$ \\
\hline$\geq 14$ & $1230(43)$ & $954(50)$ & $1378(47)$ & $3562(46)$ \\
\hline
\end{tabular}

$B P D$ Bronchopulmonary dysplasia, GA gestational age, PMA postmenstrual age.

ancludes participants with and without prior receipt of surfactant within the labeled PNA.

${ }^{\mathrm{b}}$ Includes participants who remained alive and in the hospital until for long enough to assess BPD ( $<32$ weeks GA: until 37 weeks PMA; $\geq 32$ weeks GA and hospitalized from 28 to 34 days after birth).

${ }^{\mathrm{c}}$ Excludes participants who were transferred acutely or had missing mortality data.

decreased, while late surfactant administration increased; late surfactant use in our cohort was similar to that described in the Prematurity and Respiratory Outcomes Program (PROP) cohort [18]. Although a previous study showed that beractant was the most commonly administered off-label surfactant [3], in our cohort, poractant alfa was administered the most. Preference of poractant alfa over beractant may be related to its higher phospholipid and surfactant b concentrations (key components to decrease alveolar surface tension) [19]; however, these composition differences did not result in statistically significant differences in efficacy or safety outcomes with calfactant or poractant alfa compared to beractant. Nevertheless, when stratified by GA, safety outcomes were more common for poractant alfa compared to beractant in the oldest GA group.

Overall study findings are consistent with a comparative effectiveness study of on-label surfactant that found no significant differences in air leak syndrome, BPD, or death among the three surfactants [17]; three efficacy trials that identified no significant differences in mortality or oxygen requirement at 36 weeks [20-23]; and another study, in which poractant alfa compared to beractant was associated with nonsignificant trends towards lower death and/or, BPD in infants 24-29 weeks GA [6]. In contrast to our results, prior studies identified significant benefits to poractant alfa $[6,7,9]$; but some observed outcomes appear due to differences in dosing $[9,24,25]$. Importantly, prior randomized trials have also failed to consistently demonstrate significant effect of late surfactant administration compared to placebo on clinical outcomes in infants $<33$ weeks GA $[13,26]$.

Our finding of greater frequency of safety events among infants 33-36 weeks GA administered poractant alfa compared to beractant, is consistent with a prior retrospective study that identified higher, albeit not statistically significant mortality among infants 28-32 weeks GA [27]. One potential reason for this observation could be differences in site experience in caring for more mature infants with substantial lung disease. Prior evidence for potential clinical site effects exists in a comparative effectiveness study of infants receiving on-label surfactant, where conditioning on site eliminated any difference in efficacy outcomes between surfactants [17]. Nonetheless, our GA groupspecific safety finding remained whether or not site was included in the model, suggesting that this finding was independent of potential clinical site effects.

Notably, we identified a relatively high incidence of safety events in our study population compared to estimates in prior studies and randomized trials, including the Trial of Late Surfactant for Prevention of Bronchopulmonary Dysplasia (TOLSURF), which documented transient bradycardia, desaturations, and severe respiratory decompensation in $4 \%, 12 \%$, and $<1 \%$, respectively, among infants $\leq 28$ weeks receiving late surfactant [13]. Potential differences in definitions and study population may account for differences in incidence of adverse events between studies.

The increasing administration of late surfactant over time may result from preliminary evidence suggesting that exogenous surfactant may effectively treat diseases where low lung compliance results from surfactant washout, inactivation, or dysfunction, or the suggestion that mechanical ventilation may lead to deactivation or dysfunction of surfactant [28-30]. Interestingly, most of our cohort had a diagnosis of RDS without concomitant diagnoses associated with surfactant washout, inactivation, or dysfunction (e.g., pneumonia, pulmonary hemorrhage). Since RDS presents in the first few days of life, other unidentified diagnoses, or concern that mechanical ventilation itself inactivates surfactant [31], may have been motivating factors for late surfactant administration in our cohort; more than half of our cohort was mechanically ventilated prior to late surfactant. Alternatively, prior evidence of trends toward lower incidence of death or BPD at 36 weeks' PMA [32], which may have prompted increasing late surfactant administration. The overall decrease in surfactant use since 2007 is also remarkable; based on our data, we note a substantial decrease in the proportion of extremely low birth weight infants mechanically ventilated on day of life 0 , from $90 \%$ in 1997 to $72 \%$ in 2017 . These data suggest a potential increased tendency to attempt noninvasive support as opposed to prophylactic intubation and surfactant and may support increasing use of late surfactant for rescue.

One limitation of this study was its retrospective nature, increasing the possibility for residual confounding. Additionally, some data were missing. Death and BPD diagnosis could not be determined for infants transferred to another hospital prior to 
Table 3. Safety outcomes by surfactant type ${ }^{a}$.

\begin{tabular}{|c|c|c|c|c|}
\hline GA Group Count (\%) & $\begin{array}{l}\text { Beractant } \\
\text { Total } \\
\mathbf{N}=\mathbf{2 8 9 0}\end{array}$ & $\begin{array}{l}\text { Calfactant } \\
\text { Total } \\
N=1936\end{array}$ & $\begin{array}{l}\text { Poractant alfa } \\
\text { Total } \\
N=\mathbf{2 9 7 6}\end{array}$ & $\begin{array}{l}\text { Total } \\
\mathbf{N}=\mathbf{7 8 0 2}\end{array}$ \\
\hline Hemodynamic instability & $693(24)$ & $601(31)$ & $624(21)$ & $1918(25)$ \\
\hline Respiratory deterioration & $1344 / 2783(48)$ & $998 / 1875(53)$ & $1477 / 2899(51)$ & $3819 / 7557(51)$ \\
\hline Sepsis & $99(3)$ & $100(5)$ & $91(3)$ & $290(4)$ \\
\hline Pulmonary hemorrhage & $18(1)$ & $16(1)$ & $44(1)$ & $78(1)$ \\
\hline Death within 3 days & $100 / 2816(4)$ & $64 / 1896(3)$ & $104 / 2926(4)$ & $268 / 7638(4)$ \\
\hline Composite Outcome & $1749 / 2816(62)$ & 1346/1907 (71) & $1857 / 2929(63)$ & $4952(65)$ \\
\hline
\end{tabular}

$G A$ gestational age.

ancludes infants with and without prior receipt of surfactant within the labeled PNA

discharge (Supplementary Discussion). Due to using the large, administrative database, which was primarily designed for quality improvement within the Pediatrix Medical Group, dosing of surfactants (including volume of drug administration) was unknown. We also did not have further details regarding the reasons for late surfactant administration or respiratory deterioration, the most common safety event in our cohort, or the exact timing of this deterioration relative to surfactant administration on a given day. Due to the nature of the deidentified, administrative database (where data are extracted from medical records into the database), we are not able to ask treating physicians their rationale for specific decisions. Additionally, in our main analysis we included infants with and without prior receipt of surfactant within the labeled PNA, potentially representing two populations who are physiologically different. Nonetheless, sensitivity analyses limited to the population receiving only late surfactant demonstrated similar results.

Finally, we are not certain about the reasons for increase in late surfactant use between 2000 and 2007 and subsequent leveling off; however, these changes could simply reflect variation in center practices. Despite these limitations, we believe our study is the first to compare the efficacy and safety of poractant alfa and calfactant to beractant, when administered after recommended PNAs. Additional strengths include the use of a database that allows for a sizable sample size and a large number of clinical sites, thereby potentially increasing external validity.

In our analysis of the Pediatrix BabySteps Clinical Data Warehouse, use of late surfactant in preterm infants has increased over the previous two decades. We found no evidence of overall superior efficacy or concerns about safety for poractant alfa or calfactant compared to beractant, suggesting that other factors, such as cost and availability of different surfactant preparations, may be considered. Cardiorespiratory decompensation was frequently observed following administration of all types of late surfactants. Specifically, caution may be needed when choosing to administer late poractant alfa rather than beractant in infants 33-36 weeks GA; however, additional studies are needed to verify these preliminary findings.

\section{REFERENCES}

1. Gallacher DJ, Hart K, Kotecha S. Common respiratory conditions of the newborn. Breathe. 2016;12:30-42.

2. Halliday HL. The fascinating story of surfactant. J Paediatr Child Health. 2017;53:327-32.

3. Taylor G, Jackson W, Hornik CP, Koss A, Mantena S, Homsley K, et al. Surfactant administration in preterm infants: drug development opportunities. J Pediatr. 2019;208:163-8.

4. Jeon GW. Surfactant preparations for preterm infants with respiratory distress syndrome: past, present, and future. Korean J Pediatr. 2019;62:155-61.

5. Polin RA, Carlo WA. Surfactant replacement therapy for preterm and term neonates with respiratory distress. Pediatrics. 2014;133:156-63.
6. Fujii AM, Patel SM, Allen R, Doros G, Guo CY, Testa S. Poractant alfa and beractant treatment of very premature infants with respiratory distress syndrome. J Perinatol. 2010;30:665-70.

7. Gharehbaghi MM, Sakha SH, Ghojazadeh M, Firoozi F. Complications among premature neonates treated with beractant and poractant alfa. Indian J Pediatr. 2010;77:751-4

8. Ramanathan R. Animal-derived surfactants: where are we? The evidence from randomized, controlled clinical trials. J Perinatol. 2009;29:S38-43.

9. Ramanathan R, Rasmussen MR, Gerstmann DR, Finer N, Sekar K. A randomized, multicenter masked comparison trial of poractant alfa (Curosurf) versus beractant (Survanta) in the treatment of respiratory distress syndrome in preterm infants. Am J Perinatol. 2004;21:109-19.

10. Chiesi USA, Inc. Curosurf (poractant alfa suspension) [package insert]. U.S. Food and Drug Administration website. https://www.accessdata.fda.gov/drugsatfda_docs/ label/2014/020744s028lbl.pdf. 2014. Accessed 30 June 2020.

11. ONY Biotech Inc. Infasurf-calfactant suspension [package insert]. U.S. Food and Drug Administration website. https://www.accessdata.fda.gov/drugsatfda_docs/ label/2011/020521s024lbl.pdf. 2011. Accessed 30 June 2020.

12. AbbVie Inc. Survanta-beractant suspension [package insert]. U.S. Food and Drug Administration website. https://www.accessdata.fda.gov/drugsatfda docs/label/ 2019/020032s045lbl.pdf. 2019. Accessed 30 June 2020.

13. Ballard RA, Keller RL, Black DM, Ballard PL, Merrill JD, Eichenwald EC, et al. Randomized trial of late surfactant treatment in ventilated preterm infants receiving inhaled nitric oxide. J Pediatr. 2016;168:23-9.e4.

14. Keller RL, Eichenwald EC, Hibbs AM, Rogers EE, Wai KC, Black DM, et al. The randomized, controlled trial of late surfactant: effects on respiratory outcomes at 1-year corrected age. J Pediatr. 2017;183:19-25.e2.

15. Keller RL, Merrill JD, Black DM, Steinhorn RH, Eichenwald EC, Durand DJ, et al. Late administration of surfactant replacement therapy increases surfactant protein-B content: a randomized pilot study. Pediatr Res. 2012;72:613-9.

16. Spitzer AR, Ellsbury DL, Handler D, Clark RH. The Pediatrix BabySteps Data Warehouse and the Pediatrix QualitySteps improvement project system-tools for "meaningful use" in continuous quality improvement. Clin Perinatol. 2010;37:49-70.

17. Trembath A, Hornik CP, Clark R, Smith PB, Daniels J, Laughon M. Comparative effectiveness of surfactant preparations in premature infants. J Pediatr. 2013;163:955-60.e1.

18. Greenberg JM, Poindexter BB, Shaw PA, Bellamy SL, Keller RL, Moore PE, et al. Respiratory medication use in extremely premature ( $<29$ weeks) infants during initial NICU hospitalization: Results from the prematurity and respiratory outcomes program. Pediatr Pulmonol. 2020;55:360-8.

19. Veldhuizen R, Nag K, Orgeig S, Possmayer F. The role of lipids in pulmonary surfactant. Biochim Biophys Acta. 1998;1408:90-108.

20. Attar MA, Becker MA, Dechert RE, Donn SM. Immediate changes in lung compliance following natural surfactant administration in premature infants with respiratory distress syndrome: a controlled trial. J Perinatol. 2004;24:626-30.

21. Bloom BT, Clark RH. Comparison of Infasurf (calfactant) and Survanta (beractant) in the prevention and treatment of respiratory distress syndrome. Pediatrics. 2005;116:392-9.

22. Bloom BT, Kattwinkel J, Hall RT, Delmore PM, Egan EA, Trout JR, et al. Comparison of Infasurf (calf lung surfactant extract) to Survanta (Beractant) in the treatment and prevention of respiratory distress syndrome. Pediatrics. 1997;100:31-8.

23. Logan JW, Moya FR. Animal-derived surfactants for the treatment and prevention of neonatal respiratory distress syndrome: summary of clinical trials. Ther Clin Risk Manag. 2009;5:251-60.

24. Singh N, Halliday HL, Stevens TP, Suresh G, Soll R, Rojas-Reyes MX. Comparison of animal-derived surfactants for the prevention and treatment of respiratory 
distress syndrome in preterm infants. Cochrane Database Syst Rev. 2015;12: CD010249.

25. Tridente A, De Martino L, De, Luca D. Porcine vs bovine surfactant therapy for preterm neonates with RDS: systematic review with biological plausibility and pragmatic meta-analysis of respiratory outcomes. Respir Res. 2019;20:28.

26. Hascoët J-M, Picaud J-C, Ligi I, Blanc T, Moreau F, Pinturier M-F, et al. Late surfactant administration in very preterm neonates with prolonged respiratory distress and pulmonary outcome at 1 year of age: a randomized clinical trial. JAMA Pediatr. 2016;170:365-72.

27. Paul S, Rao S, Kohan R, McMichael J, French N, Zhang G, et al. Poractant alfa versus beractant for respiratory distress syndrome in preterm infants: a retrospective cohort study. J Paediatr Child Health. 2013;49:839-44.

28. Bos AP, Tibboel D, Hazebroek FW, Molenaar JC, Lachmann B, Gommers D. Surfactant replacement therapy in high-risk congenital diaphragmatic hernia. Lancet. 1991;338:1279.

29. Deshpande S, Suryawanshi P, Ahya K, Maheshwari R, Gupta S. Surfactant therapy for early onset pneumonia in late preterm and term neonates needing mechanical ventilation. J Clin Diagn Res. 2017;11:SC09-12.

30. El Shahed Al, Dargaville PA, Ohlsson A, Soll R. Surfactant for meconium aspiration syndrome in term and late preterm infants. Cochrane Database Syst Rev. 2014;12: CD002054.

31. Merrill JD, Ballard RA, Cnaan A, Hibbs AM, Godinez Rl, Godinez MH, et al. Dysfunction of pulmonary surfactant in chronically ventilated premature infants. Pediatr Res. 2004;56:918-26.

32. Laughon M, Bose C, Moya F, Aschner J, Donn SM, Morabito C, et al. A pilot randomized, controlled trial of later treatment with a peptide-containing, synthetic surfactant for the prevention of bronchopulmonary dysplasia. Pediatrics. 2009;123:89-96.

\section{AUTHOR CONTRIBUTIONS}

Drafting, revising, and final paper preparation: $\mathrm{MDL}, \mathrm{CPH}$, and $\mathrm{KOZ}$. Oversight, funding: KOZ. Drafting, review: SK, OU, SED, RMT, AL, SB, and RHC. Initial concept, revising: $\mathrm{WMJ}, \mathrm{ML}, \mathrm{CPH}$, and KOZ. Statistical analysis: $\mathrm{CPH}$, RGG. Provided data: $\mathrm{RHC}$.

\section{FUNDING}

Supported by DCRI's R25 STAR Program (grant \#5R25HD076475-07).

\section{COMPETING INTERESTS}

MDL: research support from Duke BIGGER Program. SB: support from the National Institutes of Health, FDA, PCORI, the Rheumatology Research Foundation's Scientist Development Award, the Thrasher Research Fund, the Childhood Arthritis and Rheumatology Research Alliance, and consulting for UCB. KOZ: support from the National Institutes of Health (National Institute of Child Health and Human Development (K23 HD091398, HHSN275201000003I), the US FDA (UG3/UH3 FD 006797), the Duke Clinical and Translational Science Award (KL2TR001115-03), and industry for neonatal and pediatric drug development (www.dcri.duke.edu/research/coi.jsp). All other authors report no competing interests relevant to this paper.

\section{ETHICAL APPROVAL}

The Duke IRB approved this study with a waiver of consent.

\section{ADDITIONAL INFORMATION}

Supplementary information The online version contains supplementary material available at https://doi.org/10.1038/s41372-021-01142-2.

Correspondence and requests for materials should be addressed to K.O.Z.

Reprints and permission information is available at http://www.nature.com/ reprints

Publisher's note Springer Nature remains neutral with regard to jurisdictional claims in published maps and institutional affiliations. 